\title{
Reproducibility of cone-beam computed tomographic measurements of bone plates and the interdental septum in the anterior mandible
}

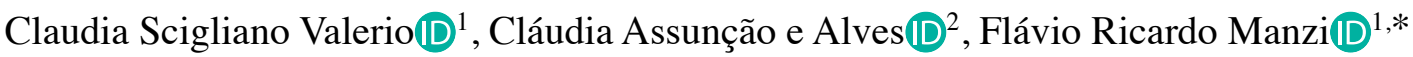 \\ ${ }^{1}$ Department of Oral Radiology, Pontifical Catholic University of Minas Gerais, Belo Horizonte, Brazil \\ ${ }^{2}$ Department of Dentistry, Pontifical Catholic University of Minas Gerais, Belo Horizonte, Brazil
}

\section{ABSTRACT}

Purpose: This study aimed to introduce a novel method to evaluate the alveolar bone and interdental septum in the anterior mandible using cone-beam computed tomography (CBCT).

Materials and Methods: Fifty-six CBCT scans from adult patients were selected. The CBCT scans were obtained before and after orthodontic treatment. The following measurements were taken: width of the alveolar bone and the interdental septum, height of the interdental septum, height of the bone plates, distance between the cementoenamel junction and marginal bone crests, and vertical positioning of the mandibular incisor, using the lingual plane as a reference. To test the reproducibility and the stability of the lingual plane, a triangle was traced in the anterior mandible. The intra-class correlation coefficient (ICC) was used to determine intra- and inter-examiner agreement. The paired Student t-test was used to evaluate the area of the triangle and the reproducibility of all measurements.

Results: The ICC was excellent for the alveolar bone and dental measurements ( 0.9989 and 0.9977 , respectively), as well as for the interdental septum (0.9987 and 0.9961 , respectively). The area of the triangles showed stability in the lingual plane $(P>0.05)$. For the alveolar bone, mandibular incisor, and interdental septum measurements, no statistically significant differences were found between the 2 examiners $(P>0.05)$, confirming the technical reliability of the measurements.

Conclusion: The method used in this study provides a valid and reproducible assessment of alveolar bone dimensions in the anterior mandible measured on CBCT images.(Imaging Sci Dent 2019; 49: 9-17)

KEY WORDS: Alveolar Process; Alveolar Bone Loss; Cone-Beam Computed Tomography; Mandible

\section{Introduction}

Methods of evaluating the alveolar bone in the anterior mandible have been an object of study in a wide range of specialties in dentistry, including orthodontics, periodontics, and implant dentistry. ${ }^{1-3}$ Orthodontic labial movement of the tooth can result in alveolar bone dehiscence or fenestration, posing a risk of gingival recession and tooth loss. ${ }^{4}$ Therefore, knowledge of the extent of vertical and

*This project was supported by the Foundation of Research Support for the State of Minas Gerais (Fundação de Amparo a Pesquisa do Estado de Minas GeraisFAPEMIG), and CAPES Foundation.

Received May 2, 2018; Revised October 16, 2018; Accepted November 18, 2018 *Correspondence to : Dr. Flávio Ricardo Manzi

Department of Oral Radiology, School of Dentistry, Pontifical Catholic University of Minas Gerais. Av. Dom José Gaspar, 500-Prédio 45, CEP: 30535-901, Belo Horizonte, Minas Gerais, Brazil

Tel) 55-31-33194341,E-mail) manzi@pucminas.br horizontal alveolar bone loss, as well as the topography of periodontal pockets, is important for accurate periodontal diagnosis and therapy. ${ }^{5}$ Dehiscence or a fenestration defect may also develop upon the placement of implants, thus requiring a bone evaluation. ${ }^{6}$

Periapical, bitewing, and panoramic radiographs are the radiographic methods most commonly used for diagnosing alveolar bone loss. However, these methods present limitations, such as image magnification, subjectivity in interpretation, 2-dimensional images of 3-dimensional structures, and reduced sensitivity in detecting changes at the levels of the facial and lingual bone crests and in detecting fenestration and alveolar bone dehiscence on the buccal and lingual side of alveolar bone. ${ }^{7,8}$ In the 1990s, the use of computed tomography (CT) and, shortly thereafter, the application of cone-beam computed tomography (CBCT) filled this void 
in radiographic diagnoses regarding the identification of the facial and lingual alveolar bones. ${ }^{3,9}$ However, a review of the literature showed that the methods used to measure the alveolar bone in the anterior mandible by means of CBCT images have not been described in an objective manner and have flaws in terms of reproducibility, which may compromise longitudinal studies.

Thus, the main challenge in radiographic studies intending to evaluate alveolar bone changes (resorption or regeneration) in the anterior mandible is to determine a stable reference that does not change in response to changes in the position of teeth due to occlusal forces, orthodontic treatment, or other dental treatments. For this reason, a reproducible and well-defined methodology must be established to evaluate bone plates. The purpose of this study, therefore, was to test the stability and reproducibility of a novel method of evaluating alveolar bone gain or loss and interdental septum changes in the anterior mandible based on CBCT images before and after orthodontic treatment.

\section{Materials and Methods}

This study was conducted after receiving approval from the local Ethics Committee on Human Research (CAAE: 30803614.3.0000.5137). Fifty-six CBCT images (28 pre-treatment, 28 post-treatment) of mandibular incisors were obtained from 28 dentate adult patients $(22.9 \pm 4.1$ years old) who presented with class I malocclusion and anterior dental crowding, and who had been referred for orthodontic treatment without extraction. Patients with gingival recession, periodontal disease, bone or soft tissue lesions in the mandibular incisor region, or no permanent mandibular incisors; patients who were smokers; and patients who used alcohol on a regular basis or who used medications that affected bone metabolism were excluded from this study.

The thickness and height of the alveolar bone and interdental septum were measured in CBCT images that had been obtained before orthodontic treatment and 3 months after the end of treatment. For CBCT scans, parameters were $80 \mathrm{kV}, 10 \mathrm{~mA}$, and $12 \mathrm{~s}$ of exposure, and the scans were acquired with $0.076-\mathrm{mm}$ isotropic voxel size and a small field of view (FOV) (Kodak 9000 3D Cone Beam CT; East Kodak Company, Rochester, NY, USA). Linear and angular measurements from the CBCT images were obtained using the CS 3D Imaging software (Kodak Dental Systems, Rochester, NY, USA). The thickness and height of the alveolar bone and interdental septum were measured to the nearest $0.076 \mathrm{~mm}$.

All of the alveolar bone measurements were performed in the parasagittal slices of the mandibular incisors that passed through the center of the mesial-distal width of the root, following the long dental axis. Measurements of the interdental septa were also performed in the parasagittal slices that passed through the center of the bone septum (Fig. 1). The benchmarks used in the present study and their definitions are presented in Table 1.

\section{Tracing the lingual plane}

The lingual canal was chosen as the initial reference point to obtain the measurements of interest. First, the lingual foramen and the lingual canal were located. Next, a

Table 1. Description of variables

\begin{tabular}{ll}
\hline \multicolumn{1}{c}{ Variables } & \multicolumn{1}{c}{ Description } \\
\hline Alveolar bone and mandibular incisor & \\
A1 & Facial lingual thickness of alveolar bone $3 \mathrm{~mm}$ below the CEJ line \\
A2 & Facial lingual thickness of alveolar bone $6 \mathrm{~mm}$ below the CEJ line \\
A3 & Facial lingual thickness of alveolar bone $9 \mathrm{~mm}$ below the CEJ line \\
A4 & Facial lingual thickness of alveolar bone $12 \mathrm{~mm}$ below the CEJ line \\
FH-MBC-LP & Facial height of the alveolar ridge from the facial marginal bone crest to the lingual plane \\
LH-MBC-LP & Lingual height of the alveolar ridge from the lingual marginal bone crest to the lingual plane \\
F-CEJ-MBC & Distance from the facial CEJ to the facial marginal bone crest \\
L-CEJ-MBC & Distance from the lingual CEJ to the lingual marginal bone crest \\
MI & Pulp chamber ceiling of the mandibular incisor to the lingual plane \\
Interdental septum & \\
SH & Bone crest height of the interdental septum \\
S1 & Alveolar ridge thickness of the interdental septum 3 mm below the bone crest \\
S2 & Alveolar ridge thickness of the interdental septum 6 mm below the bone crest \\
S3 & Alveolar ridge thickness of the interdental septum 9 mm below the bone crest \\
S4 & Alveolar ridge thickness of the interdental septum $12 \mathrm{~mm}$ below the bone crest \\
\hline
\end{tabular}

CEJ: cementoenamel junction 
line was traced over the lingual canal, beginning at the lingual foramen and extending in the direction of the facial limit of the symphysis. In this study, this line traced over the lingual canal was referred to as the lingual plane (Fig. 2). After the lingual plane had been outlined in a tomographic section of the sagittal plane, the software enabled it to be

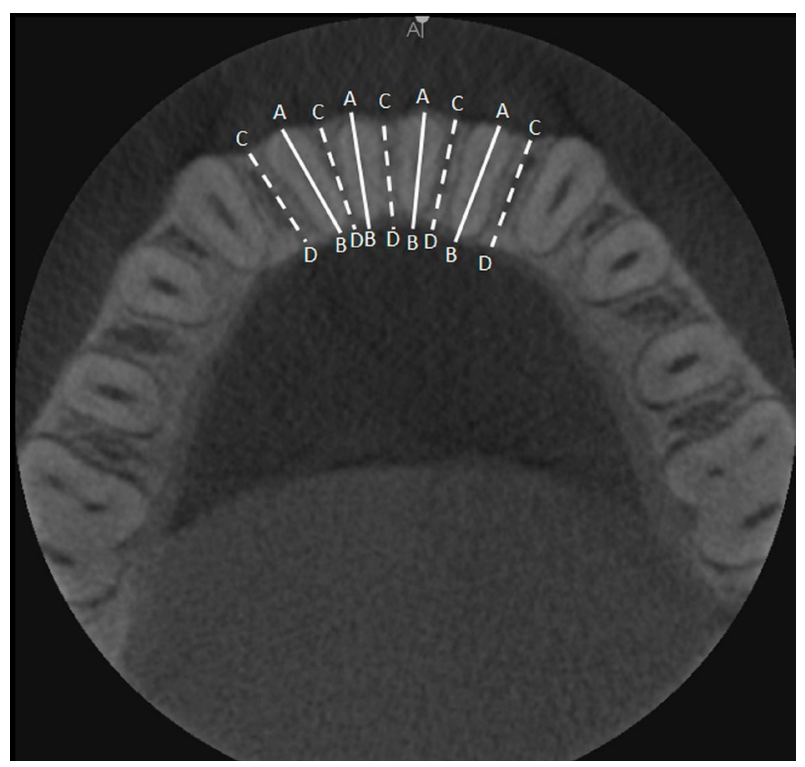

Fig. 1. On the axial slice of the mandible, the localization of the parasagittal slices was determined for the measurements of the alveolar bone (continuous AB lines) and the measurements of the interdental septum (interrupted CD lines). viewed in all parasagittal sections.

Two aspects must be considered when tracing the lingual plane: the ramification and the inclination of the lingual canal. According to Babiuc et al., ${ }^{10}$ some patients (12.1\%) have multiple canals; therefore, when many canals are present, one must consider the lingual canal to be that with the longest extension. As regards the trajectory of the lingual canal in the sagittal plane, some patients present a rather steep route. In such cases, a compensatory plane with a $20^{\circ}$ reduction in the slope of the lingual plane should be traced in such a way as to intercept the lingual foramen and facilitate the measurement of the height of the bone plates (Fig. 3 ). This information must be recorded in the first measurement and repeated in the final measurement when evaluating the same structures of interest in longitudinal studies.

\section{Tracing the triangle}

To test the reproducibility and the stability of the lingual plane between times T1 (pre-treatment) and T2 (post-treatment), a triangle was traced in the anterior region of the mandible in the median sagittal plane. The lingual plane constituted the first side of this triangle (L1). Next, a line was traced with a $20^{\circ}$ slope from the facial extremity of the lingual plane, extending to the lingual limit of the symphysis (L2). A third line was traced, joining the lingual extremity of the 2 first lines (L3). To calculate the area of this triangle, the height of the triangle was traced (h) (Fig. 4).
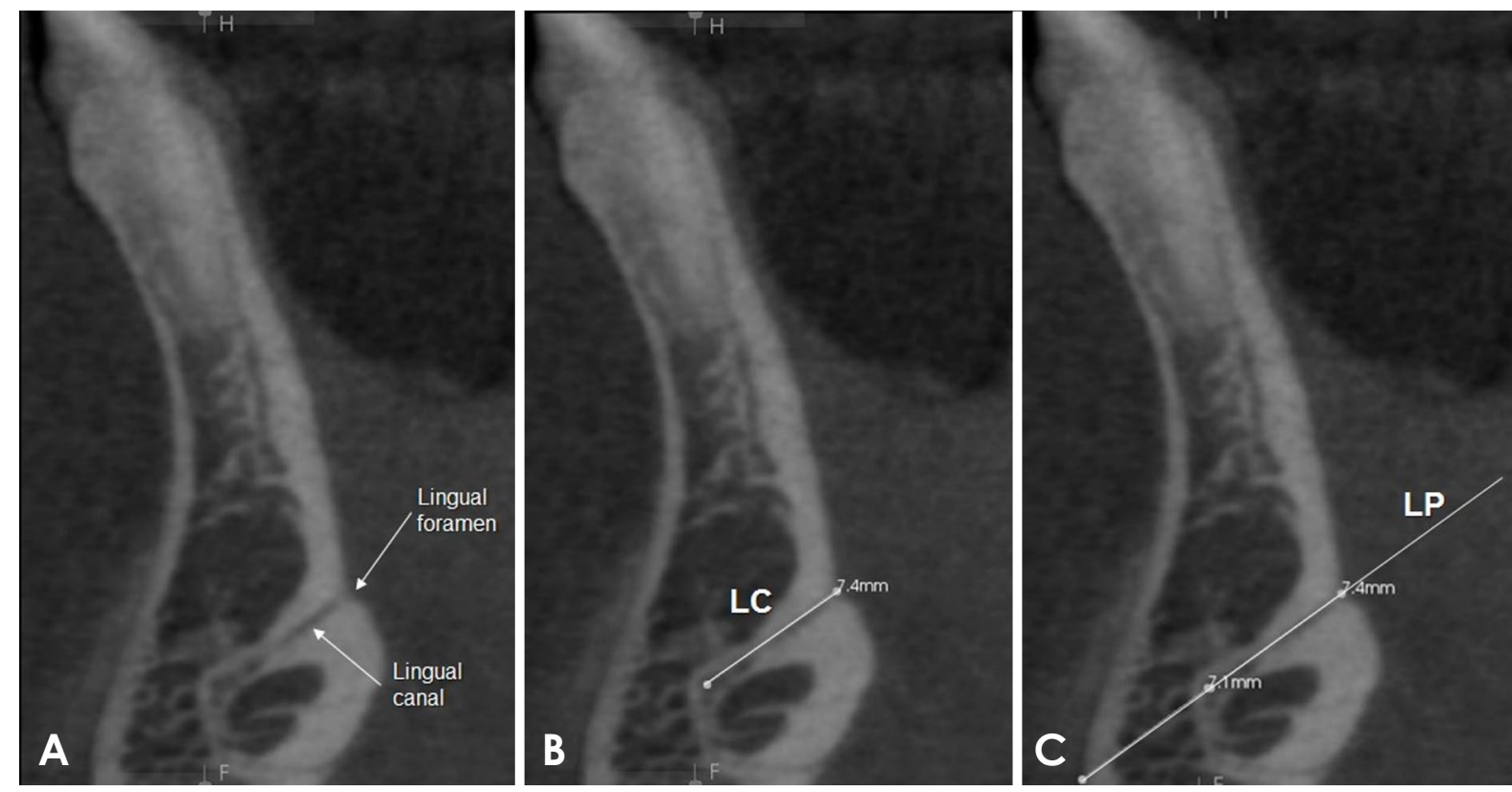

Fig. 2. Parasagittal slice. A. Localization of the lingual foramen and the mandibular lingual canal. B. Tracing of the mandibular lingual canal (LC). C. Extension of the lingual canal line to obtain the lingual plane (LP). 


\section{Measurement of the height of the facial and lingual bone plates}

The measurements of the height of the facial and lingual bone plates were obtained in millimeters, beginning with the highest point of the marginal bone crest (MBC) and moving in the direction of the lingual plane in order to intercept the lingual plane perpendicularly. The measurements of these lines represented the facial height of the al-

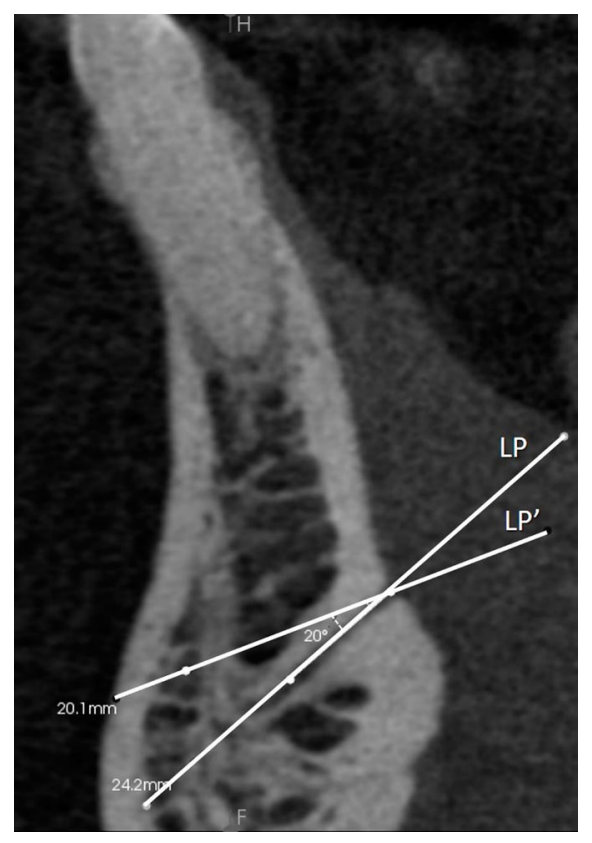

Fig. 3. Parasagittal slice. Tracing of the compensatory lingual plane $\left(\mathrm{LP}^{\prime}\right)$, with $20^{\circ}$ of compensation. LP: lingual plane. veolar ridge from the facial $\mathrm{MBC}$ to the lingual plane $(\mathrm{FH}-$ MBC-LP) and the lingual height of the alveolar ridge from the lingual MBC to the lingual plane (LH-MBC-LP) (Fig. $5 \mathrm{~A})$. The distances between the lingual cementoenamel

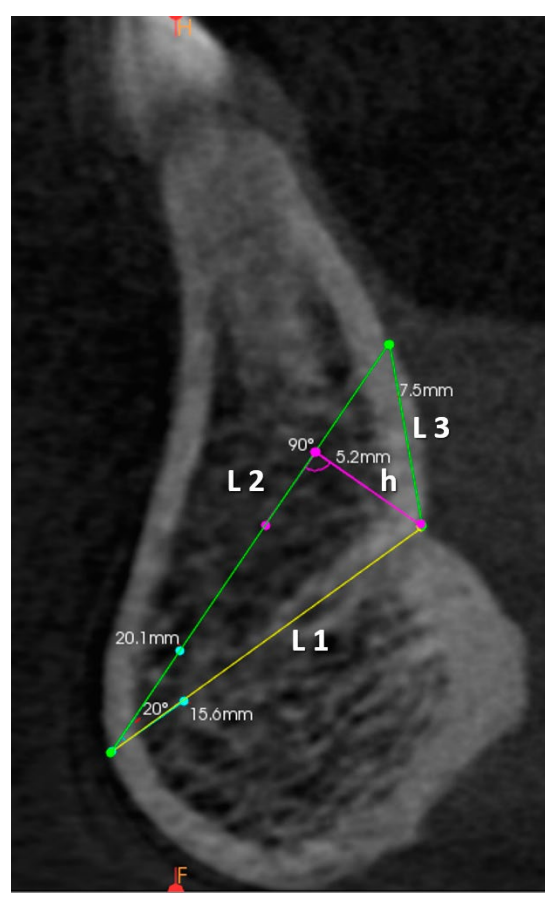

Fig. 4. Parasagittal slice. Tracing of the triangle. First, line 1 was traced over the lingual plane, respecting the limits of the symphysis (L1). The second side was traced with an internal angle of $20^{\circ}$, beginning from the facial limit of L1 (L2). The third side joined the lingual extremities of sides L1 and L2, forming L3. Height of the triangle $(\mathrm{h})$.
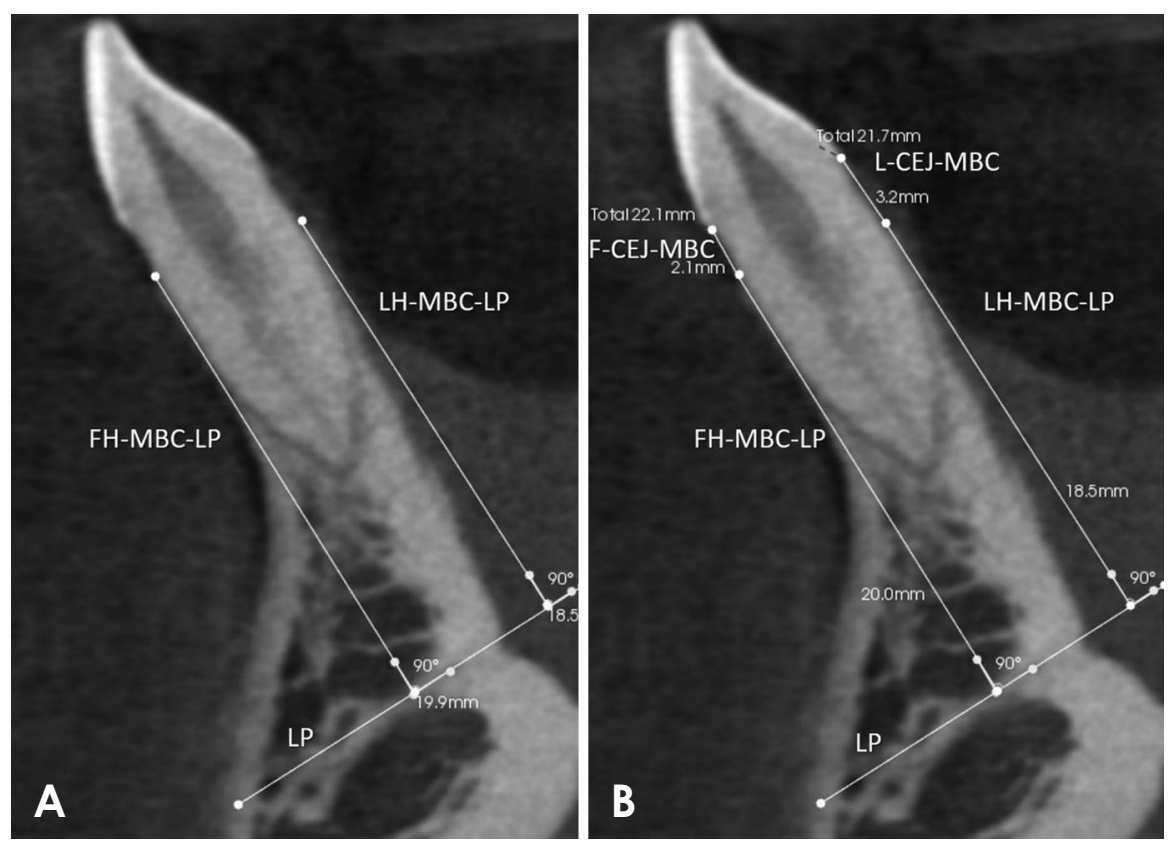

Fig. 5. Parasagittal slice. A. Measurement of the height of the facial bone plate $(\mathrm{FH}-$ MBC-LP) and the lingual bone plate ( $\mathrm{LH}-$ MBC-LP). B. Measurement of the distance between the $\mathrm{CEJ}$ and the marginal bone crest on the facial side (F-CEJ-MBC) and on the lingual side (L-CEJ-MBC). MBC: marginal bone crest; LP: lingual plane. 

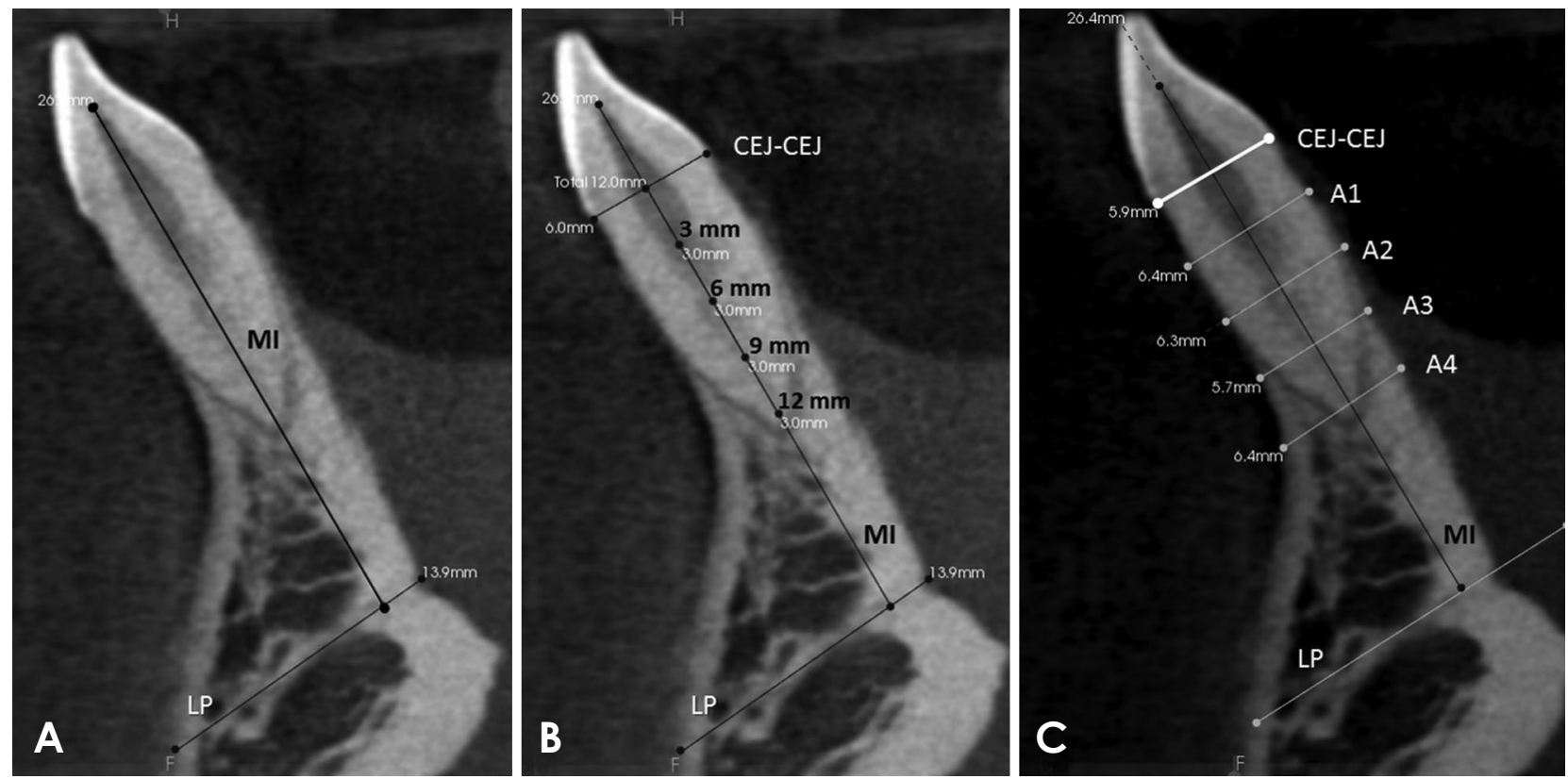

Fig. 6. Parasagittal slice. A. Measurement of the vertical positioning of the mandibular incisor (MI line). B and C. Measurement of the thickness of the alveolar bone. Initially, a line was traced along the incisor's long axis (MI line) and the joining line between the facial and lingual CEJs (CEJ-CEJ line). Next, the thicknesses of the alveolar process were measured using the A1, A2, A3, and A4 lines, traced 3 mm, $6 \mathrm{~mm}, 9 \mathrm{~mm}$, and $12 \mathrm{~mm}$ below the CEJ-CEJ line, respectively.

junction (CEJ) and the lingual MBC (L-CEJ-MBC) and between the facial CEJ and the facial MBC (F-CEJ-MBC) were also recorded (Fig. 5B).

\section{Measurement of the thickness of the alveolar bone}

First, in the parasagittal cut, the long axis of the mandibular incisor was traced from the pulp chamber ceiling to the lingual plane, following the center of the dental canal (mandibular incisor [MI] line) (Fig. 6A). Next, a line joining the facial and the lingual CEJ was drawn (CEJ-CEJ line). The measurements of the thickness of the alveolar bone thickness in the alveolar process were obtained in millimeters based on the lines traced parallel to the lingual plane, which were drawn beginning at the intersection between the MI and CEJ-CEJ lines, at points located 3, 6, 9, and 12 mm below the CEJ-CEJ line (Fig. 6B). In this manner, the thicknesses A1, A2, A3, and A4 were obtained, located 3, 6,9 , and $12 \mathrm{~mm}$ below the CEJ-CEJ line, respectively (Fig. $6 \mathrm{C})$.

\section{Measurement of the height and thickness of the interdental septum}

The lingual plane can also be used as a reference to evaluate the interdental septum. Much like the alveolar bone region, here one can also measure the height and thickness of the interdental bone septum through the parasagittal plane.
To measure the height of the interdental septum, the sagittal tomography slice was placed in the center of the interdental space. Beginning at the highest point of the ridgethe bone crest-a line was drawn perpendicular to the lingual plane. Along this line, the distance was measured between the bone crest and the lingual plane, reaching the height of the interdental septum. This line was referred to as the bone crest height of the interdental septum (SH) (Fig. 7A).

The measurement of the thickness of the interdental septum used the SH line as the reference point. Beginning from the bone crest in the direction of the lingual plane, 4 points were marked to measure the thickness of the interdental ridge, at $3 \mathrm{~mm}, 6 \mathrm{~mm}, 9 \mathrm{~mm}$, and $12 \mathrm{~mm}$ below the bone crest. Next, parallel lines were drawn to the lingual plane, passing through the 4 points cited above, which extended from the facial surface to the lingual surface of the septum in order to measure the thickness of the alveolar bone. Thus, the thicknesses S1, S2, S3, and S4 were obtained, located 3, 6, 9, and $12 \mathrm{~mm}$ below the bone crest, respectively (Fig. 7B).

\section{Statistical analyses}

Two observers -1 orthodontist and 1 oral radiologist-were calibrated. All measurements were taken twice, with a time interval of 1 week between each measurement. 

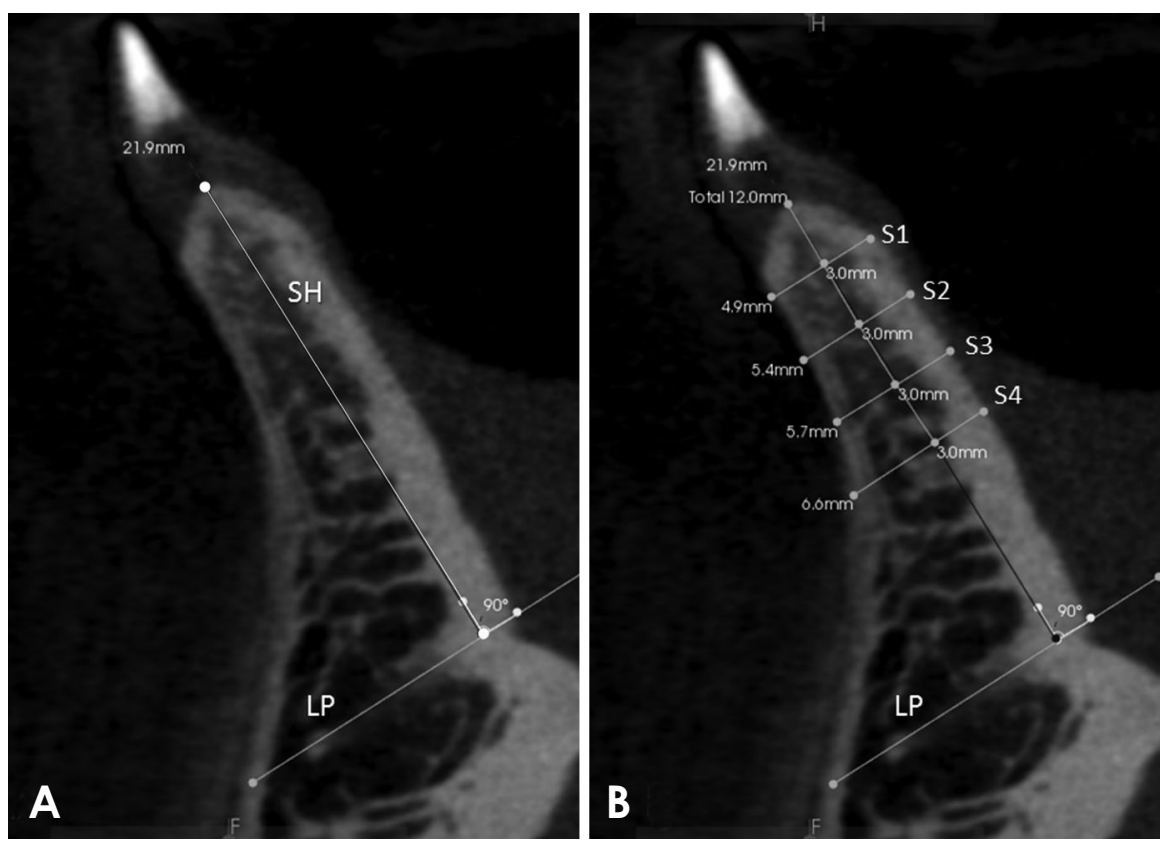

Fig. 7. Parasagittal slice. A. Measurement of the height of the interdental septum through the $\mathrm{SH}$ line, traced perpendicular to the lingual plane (LP). B. Measurement of the thickness of the interdental septum at points located $3 \mathrm{~mm}(\mathrm{~S} 1), 6 \mathrm{~mm}(\mathrm{~S} 2), 9$ $\mathrm{mm}(\mathrm{S} 3)$, and $12 \mathrm{~mm}(\mathrm{~S} 4)$ below the bone crest. LP: lingual plane, $\mathrm{SH}$ : bone crest height.

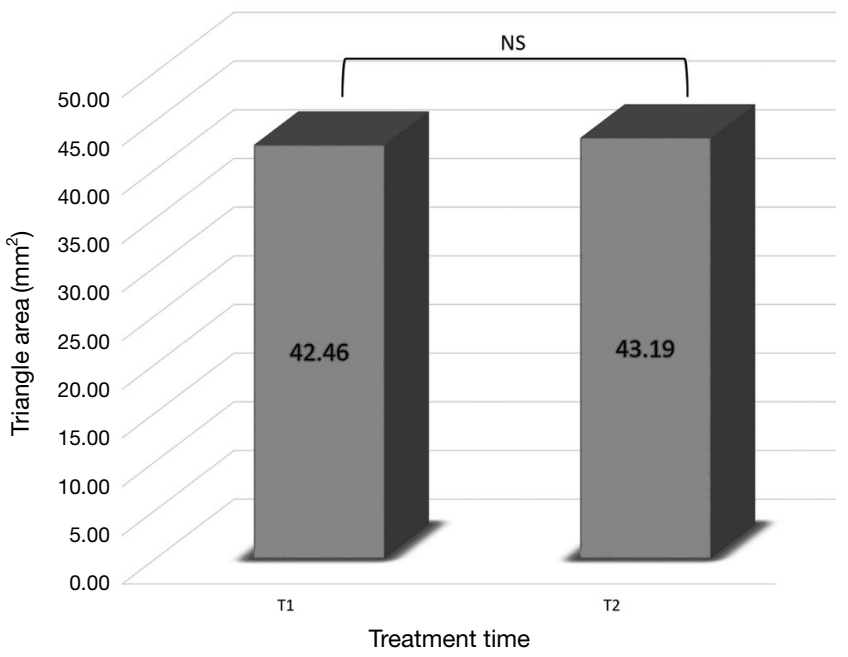

Fig. 8. Triangle areas obtained at time 1 (T1) and time 2 (T2) (NS: $P>0.05)$.

The intra-class correlation coefficient was used to determine intra- and inter-examiner agreement (ICC). ${ }^{11}$ The paired Student t-test was used to evaluate the area of the triangle, as well as the alveolar bone, mandibular incisor, and interdental septum measurements. Random error was quantified using the Dahlberg formula. ${ }^{12}$

\section{Results}

The area of the triangle was used to test the stability of the lingual plane. Intra- and inter-examiner agreement was
Table 2. Intra-class correlation coefficients for the intra- and inter-examiner agreement of the measurements

\begin{tabular}{lccc}
\hline & Triangle & Alveolar bone & Interdental septum \\
\hline Intra-examiner & 0.9185 & 0.9989 & 0.9987 \\
Inter-examiner & 0.8606 & 0.9977 & 0.9961 \\
\hline
\end{tabular}

Table 3. Dahlberg error test (mm)

\begin{tabular}{lc}
\hline Variables & Dahlberg \\
\hline A1 & 0.17 \\
A2 & 0.14 \\
A3 & 0.13 \\
A4 & 0.15 \\
FH-MBC-LP & 0.28 \\
LH-MBC-LP & 0.29 \\
F-CEJ-MBC & 0.15 \\
L-CEJ-MBC & 0.16 \\
MI & 0.20 \\
SH & 0.26 \\
S1 & 0.14 \\
S2 & 0.15 \\
S3 & 0.15 \\
S4 & 0.13 \\
\hline
\end{tabular}

excellent for the area of the triangle $(0.9185$ and 0.8606 , respectively). The paired Student t-test showed no statistically significant difference among the measurements of the area of the triangle when times $\mathrm{T} 1$ and $\mathrm{T} 2$ were compared $(P>0.05)$ (Fig. 8).

Intra- and inter-examiner agreement was also excellent 
Table 4. Descriptive statistics for the variables. Differences between examiner 1 and examiner 2 (paired Student t-test)

\begin{tabular}{lrrr}
\hline Variables & Examiner 1 & Examiner 2 & 95\% CI \\
& Mean \pm SD & Mean \pm SD & \\
\hline Alveolar bone and mandibular incisor & & -0.05 to 0.14 \\
A1 & $6.78 \pm 0.35$ & $6.73 \pm 0.38$ & -0.16 to 0.11 \\
A2 & $6.98 \pm 0.42$ & $7.01 \pm 0.42$ & -0.25 to 0.01 \\
A3 & $7.46 \pm 1.00$ & $7.58 \pm 1.10$ & -0.30 to 0.02 \\
A4 & $8.54 \pm 1.70$ & $8.68 \pm 1.73$ & -0.09 to 0.33 \\
FH-MBC-LP & $19.56 \pm 2.33$ & $19.44 \pm 2.30$ & -0.02 to 0.08 \\
LH-MBC-LP & $18.67 \pm 2.40$ & $18.64 \pm 2.41$ & -0.16 to 0.21 \\
F-CEJ-MBC & $1.89 \pm 0.67$ & $1.86 \pm 0.64$ & -0.06 to 0.16 \\
L-CEJ-MBC & $2.23 \pm 1.11$ & $2.18 \pm 1.07$ & -0.31 to 0.13 \\
MI & $25.97 \pm 2.36$ & $26.06 \pm 2.20$ & 0.67 \\
Interdental septum & & & 0.07 \\
SH & $21.31 \pm 2.71$ & $21.44 \pm 2.32$ & -0.44 to 0.17 \\
S1 & $6.25 \pm 0.49$ & $6.13 \pm 0.36$ & -0.03 to 0.26 \\
S2 & $6.64 \pm 0.64$ & $6.66 \pm 0.70$ & -0.16 to 0.11 \\
S3 & $7.38 \pm 0.87$ & $7.40 \pm 0.87$ & -0.03 to 0.00 \\
S4 & $8.26 \pm 1.38$ & $8.30 \pm 1.42$ & -0.09 to 0.00 \\
\hline
\end{tabular}

CI: confidence interval

for the alveolar bone and interdental septum measurements. For the alveolar bone, intra- and inter-examiner agreement was 0.9989 and 0.9977 , respectively, while for the interdental septum the agreement values were 0.9987 and 0.9961 , respectively (Table 2).

The random method error ranged from 0.13 to 0.29 for all variables (Table 3 ). Table 4 shows the mean and standard deviation of the alveolar bone, mandibular incisor, and interdental septum measurements, together with the results of the inter-examiner analysis. No statistically significant difference was found between the 2 examiners $(P>0.05)$, showing the technical reliability of the measurements. Descriptive statistics also showed that the first and second variables of alveolar bone thickness (A1 and A2) were similar, with an increase in thickness as the most apical region was evaluated. The same pattern was observed for the variables $\mathrm{S} 1$ and $\mathrm{S} 2$ for the interdental septum.

\section{Discussion}

Orthodontic tooth movement can cause the root to brush up against the cortical bone, causing dehiscence and fenestration. In this context, it is important for orthodontists to observe the facial and lingual bone plates, mainly in adult patients, because they may also present some degree of alveolar bone loss before orthodontic treatment is started. CBCT is an imaging method that can access the lingual and facial aspects of dental roots without superposition of anatomic structures; however, a reproducible and well-de- fined methodology for measuring the alveolar bone plate on CBCT images has not yet been established. For this reason, this study sought to introduce a novel and reproducible method to evaluate the alveolar bone and interdental septum in the anterior mandible. The use of the lingual canal as a stable reference has not previously been investigated.

The first reference for the measurement of a structure is the starting point from which all other measurements will be performed and repeated. Thus, it is very important that this reference point can be localized in a precise and reproducible way to serve as a solid basis for future studies. ${ }^{13}$ Prior studies have utilized first reference points located in structures that can change with orthodontic treatment, such as the alveolar crest, the root apex, or the incisal and occlusal surface, or those that contain a complex system of localization, such as the coordinate system used to trace the mandibular plane; therefore, the use of these reference points has led to insufficient or variable replicability of the majority of findings. ${ }^{2,14-18}$

The lingual canal presents distinct radiopaque walls, which are easily found in the median region of the mandible. The number of lingual foramina and canals can vary from patient to patient. In the findings of Babiuc et al., ${ }^{10} 71.9 \%$ of the analyzed cases presented a canal, while $28.1 \%$ of the cases presented more than 1 foramen and canal. In the present study, when multiple canals were found, the lingual canal proved to have a more extensive trajectory.

The lingual canal trajectory can also vary when observed 
in the sagittal plane. One tomographic study of the anatomy of the lingual canal reported that $62 \%$ of canals had a descending trajectory, $17.3 \%$ had an anterior trajectory, and $20.7 \%$ had an ascending trajectory. ${ }^{10}$ In the present study, all patients had a lingual canal with a descending trajectory; however, when the lingual canal trajectory was highly sloped (that is, heavily inclined in the vertical direction), a compensatory lingual plane was traced, beginning from the lingual foramen. We observed that the introduction of this stage did not reduce the reproducibility of the method, as it was necessary to record the number of degrees of compensation adopted in the first measurement of the CBCT images so that the examiner could replicate this measure in the line traced to highlight the compensatory lingual plane of the second measurement.

Prior studies have verified the need to evaluate the vertical movement of the lower incisors, in addition to the movements of rotation, translation, and proclination or retroclination of the incisor, in the analysis of vertical bone loss detected by the increase in the distance between the bone crest and the CEJ. ${ }^{1,19}$ With this new method, it is possible to register the vertical positioning of the incisors, allowing for the evaluation of vertical changes in the intrusion and extrusion of the mandibular incisors during orthodontic treatment. For this, it is only necessary to measure the distance from the pulp chamber ceiling to the lingual plane, through the line of the long dental axis (MI line).

The evaluation of the interdental septum compliments the analysis of the evaluation of the facial and lingual bone plates, since all of the alveolar walls must be evaluated as regards bone loss or gain. The intra- and inter-examiner agreement values obtained in this study were 0.9987 and 0.9961 , respectively, showing excellent agreement. Hence, our results show that this method can also be used in research that seeks to evaluate bone height in the interdental region. Intra- and inter-examiner agreement for the evaluation of the alveolar bone was also excellent, confirming the excellent reproducibility of this method, which will be useful in transversal and longitudinal studies that seek to evaluate the bone anatomy of the mandibular incisor region.

The stability of the lingual plane between time points $\mathrm{T} 1$ and T2 was evaluated through the area of the triangle. There were no statistically significant differences among the measurements of the area of the triangle when time points $\mathrm{T} 1$ and $\mathrm{T} 2$ were compared $(P>0.05)$, proving that the lingual plane did not change with orthodontic treatment. This finding may well have resulted from this anatomical structure being located in the basal bone (that is, below the dental roots), and thus not being affected by the orthodontic movement of the teeth. In contrast, vertical and horizontal changes in the symphysis during facial growth can occur; ${ }^{20,21}$ therefore, we do not recommend the use of this methodology in patients who have not completed pubertal growth.

The Student t-test showed no statistically significant difference in the inter-examiner measurements, which proved to show adequate reproducibility in the measurement of the variables chosen for the study $(P>0.05)$ (Table 4$)$. The ICC values showing intra- and inter-examiner agreement were excellent for all variables, supporting the appropriateness of the method. Previous studies that evaluated the reliability of buccal bone height and thickness measurements from CBCT also showed high inter-rater reliability in measurements (0.76-0.99); however, the variables used had different reference points from the current study. ${ }^{22,23}$

This study used a CBCT device with a relatively small voxel size and small FOV. When the bone thickness is similar to, or smaller than, the voxel size, the distance between the CEJ and the MBC can be overestimated; thus, decreasing the voxel size can improve the measurement accuracy. $^{22,24,25}$ Considering this limitation, we recommend that CBCT images be acquired on devices that use small voxels and a small FOV to better view the alveolar bone plates.

In conclusion, the method used in this study provides a valid and reproducible evaluation of the dimensions of the alveolar process in the anterior mandible using CBCT. This method can be used in cross-sectional studies and in longitudinal research seeking to evaluate changes in facial and lingual bone plates, vertical and angular changes of the mandibular incisors, and changes in the interdental septum by means of CBCT images. Thus, this can be a good measurement method for the anterior mandible for anthropologists, forensic experts, anatomists, orthodontists, and surgeons.

\section{References}

1. Garlock DT, Buschang PH, Araujo EA, Behrents RG, Kim KB. Evaluation of marginal alveolar bone in the anterior mandible with pretreatment and posttreatment computed tomography in nonextraction patients. Am J Orthod Dentofacial Orthop 2016; 149: 192-201.

2. Sarikaya S, Haydar B, Ciğer S, Ariyürek M. Changes in alveolar bone thickness due to retraction of anterior teeth. Am J Orthod Dentofacial Orthop 2002; 122: 15-26.

3. Fuhrmann R. Three-dimensional interpretation of labiolingual bone width of the lower incisors. Part II. J Orofac Orthop 1996; 57: 168-85.

4. Pini-Prato G, Nieri M, Pagliaro U, Giorgi TS, La Marca M, Franceschi D, et al. Surgical treatment of single gingival reces- 
sions: clinical guidelines. Eur J Oral Implantol 2014; 7: 9-43.

5. Fuhrmann RA, Wehrbein H, Langen HJ, Diedrich PR. Assessment of the dentate alveolar process with high resolution computed tomography. Dentomaxillofac Radiol 1995; 24: 50-4.

6. Merli M, Merli I, Raffaelli E, Pagliaro U, Nastri L, Nieri M. Bone augmentation at implant dehiscences and fenestrations. A systematic review of randomised controlled trials. Eur J Oral Implantol 2016; 9: 11-32.

7. Takeshita WM, Vessoni Iwaki LC, Da Silva MC, Tonin RH. Evaluation of diagnostic accuracy of conventional and digital periapical radiography, panoramic radiography, and cone-beam computed tomography in the assessment of alveolar bone loss. Contemp Clin Dent 2014; 5: 318-23.

8. Kumar V, Ludlow J, Soares Cevidanes LH, Mol A. In vivo comparison of conventional and cone beam CT synthesized cephalograms. Angle Orthod 2008; 78: 873-9.

9. Mozzo P, Procacci C, Tacconi A, Martini PT, Andreis IA. A new volumetric CT machine for dental imaging based on the conebeam technique: preliminary results. Eur Radiol 1998; 8: 155864.

10. Babiuc I, Tărlungeanu I, Păuna M. Cone beam computed tomography observations of the lingual foramina and their bony canals in the median region of the mandible. Rom J Morphol Embryol 2011; 52: 827-9.

11. Shrout PE, Fleiss JL. Intraclass correlations: uses in assessing rater reliability. Psychol Bull 1979; 86: 420-8.

12. Dahlberg G. Standard error and medicine. Acta Genet Stat Med 1949; 1: 313-21.

13. Begley CG, Ioannidis JP. Reproducibility in science: improving the standard for basic and preclinical research. Circ Res 2015; 116: 116-26.

14. Bimstein E, Crevoisier RA, King DL. Changes in the morphology of the buccal alveolar bone of protruded mandibular permanent incisors secondary to orthodontic alignment. Am J Orthod Dentofacial Orthop 1990; 97: 427-30.

15. Qu X, Liu Z, Wang Y, Fang Y, Du M, He H. Dentofacial traits in association with lower incisor alveolar cancellous bone thickness: a multiple regression analysis. Angle Orthod 2017; 87: 409-15.
16. Baysal A, Uysal T, Veli I, Ozer T, Karadede I, Hekimoglu S. Evaluation of alveolar bone loss following rapid maxillary expansion using cone-beam computed tomography. Korean $\mathbf{J}$ Orthod 2013; 43: 83-95.

17. Garib DG, Menezes MH, Silva Filho OG, Santos PB. Immediate periodontal bone plate changes induced by rapid maxillary expansion in the early mixed dentition: CT findings. Dental Press J Orthod 2014; 19: 36-43.

18. Dudic A, Giannopoulou C, Leuzinger M, Kiliaridis S. Detection of apical root resorption after orthodontic treatment by using panoramic radiography and cone-beam computed tomography of super-high resolution. Am J Orthod Dentofacial Orthop 2009; 135: 434-7.

19. Yared KF, Zenobio EG, Pacheco W. Periodontal status of mandibular central incisors after orthodontic proclination in adults. Am J Orthod Dentofacial Orthop 2006; 130: 6.e1-8.

20. Buschang PH, Julien K, Sachdeva R, Demirjian A. Childhood and pubertal growth changes of the human symphysis. Angle Orthod 1992; 62: 203-10.

21. Aki T, Nanda RS, Currier GF, Nanda SK. Assessment of symphysis morphology as a predictor of the direction of mandibular growth. Am J Orthod Dentofacial Orthop 1994; 106: 60-9.

22. Sun Z, Smith T, Kortam S, Kim DG, Tee BC, Fields H. Effect of bone thickness on alveolar bone-height measurements from cone-beam computed tomography images. Am J Orthod Dentofacial Orthop 2011; 139: e117-27.

23. Timock AM, Cook V, McDonald T, Leo MC, Crowe J, Benninger BL, et al. Accuracy and reliability of buccal bone height and thickness measurements from cone-beam computed tomography imaging. Am J Orthod Dentofacial Orthop 2011; 140 : 734-44.

24. Leung CC, Palomo L, Griffith R, Hans MG. Accuracy and reliability of cone-beam computed tomography for measuring alveolar bone height and detecting bony dehiscences and fenestrations. Am J Orthod Dentofacial Orthop 2010; 137: S109-19.

25. Lund H, Gröndahl K, Gröndahl HG. Cone beam computed tomography evaluations of marginal alveolar bone before and after orthodontic treatment combined with premolar extractions. Eur J Oral Sci 2012; 120: 201-11. 